\title{
On a System of Nonlinear Optical Wave Equations in Two-Spatial Dimension
}

\author{
Jeng-Eng $\operatorname{Lin}^{1}$ \\ ${ }^{1}$ Department of Mathematics and Statistics, Georgetown University, U. S. A. \\ Correspondence: Jeng-Eng Lin, Address. Department of Mathematics and Statistics, Georgetown University, \\ Washington, DC 20057, U. S. A.
}

Received: January 22, 2018 Accepted: February 9, 2018 Online Published: March 12, 2018

doi:10.5539/jmr.v10n2p100 URL: https://doi.org/10.5539/jmr.v10n2p100

\begin{abstract}
We show that for a system of nonlinear weakly dispersive wave equations in two-spatial dimension, which is a model in nonlinear optics, the local $\mathrm{L}^{2}$ norm of its solutions decays to zero as time approaches infinity.
\end{abstract}

Keywords: Decay, optical wave, two-dimensional

\section{Introduction}

The equation

$$
u_{t}-u_{x}+u_{x x x}+\mathrm{i}|u|^{2} \mathrm{u}=0
$$

which describes a model of nonlinear modulated dispersive wave was first discovered to have a very important application in optical communication by Wai, Menyuk, Lee, \& Chen (1986), Wai, Menyuk, Chen, \& Lee (1987), and Wai, Chen, \& Lee (1990).

In this paper, we will discuss the asymptotic behavior of the wave of a weakly dispersive medium, which is described by the two-dimensional generalization of the equation (1.1). The wave is still propagating in the $\mathrm{x}$-direction but with a weakly perturbation in the $y$-direction.

$$
\begin{gathered}
u_{t}-u_{x}+u_{x x x}+\mathrm{i}|u|^{2} \mathrm{u}=-w_{y}, \\
w_{x}=u_{y}
\end{gathered}
$$

We will show that the smooth solutions decay in time in the local $L^{2}$ norm. The motivation to study this system of equations is similar to the study of the well-known Kadomtsev-Petviashivili equation (Kadomtsev \& Petviashvil 1970)

$$
\begin{gathered}
u_{t}+6 \mathrm{u} u_{x}+u_{x x x}=-w_{y} \\
w_{x}=u_{y}
\end{gathered}
$$

to the well-known Korteweg-de Vries equation (Korteweg \& de Vries 1895)

$$
u_{t}+6 \mathrm{u} u_{x}+u_{x x x}=0 \text {. }
$$

This paper is the first paper to study the proposed system of equations (1.2 a) \& (1.2 b).

\section{Two Conservation Laws}

Conservation laws are very important in the physics. The famous Noether's Theorem (Noether 1918)

states that every invariance of motion of a physical system has a corresponding conservation law. Furthermore, every conservation law is a constraint to the governing system of equations of the physical system. Here we present two conservation laws of (1.2 a) and (1.2b).

Multiplying both sides of equations (1.2.a) and (1.2.b) by $u^{*}$, where $u^{*}$ is the complex conjugate of $\mathrm{u}$, and then taking the real part of the equation, we get

$$
\partial\left((|u|)^{2}\right) / \partial \mathrm{t}=\partial \mathrm{C} / \partial \mathrm{x}+\partial \mathrm{D} / \partial \mathrm{y}
$$

where $\left.\mathrm{C}=|u|^{2}-(|u|)^{2}\right)_{x x}+3\left|u_{x}\right|^{2}+|u|^{2}$ and $\mathrm{D}=-\mathrm{w} u^{*}-w^{*} \mathrm{u}$

Thus 


$$
\iint_{R^{2}}|u|^{2}(x, y, t) d x d y=\text { constant } .
$$

where $w^{*}$ is the complex conjugate of $\mathrm{w}$.

Another conservation law can be derived as

$$
\partial\left(u^{*} u_{x}-u u_{x}^{*}\right) / \partial \mathrm{t}=\partial \mathrm{E} / \partial \mathrm{x}+\partial \mathrm{F} / \partial \mathrm{y}
$$

where $\mathrm{E}=-u_{x x}^{*} u_{x}+u_{x x} u_{x}^{*}-\mathrm{i}|u|^{4}-w_{y}^{*} \mathrm{u}+w_{y} u^{*}+u^{*} u_{x}-u u_{x}^{*}-u^{*} u_{x x x}+u u_{x x x}^{*}+u_{x}^{*} u_{x x}-u_{x} u_{x x}^{*}$ and $\mathrm{F}=\mathrm{u}^{*}{ }_{\mathrm{y}} \mathrm{u}-\mathrm{u}_{y} \mathrm{u}^{*}$ $-\mathrm{u}^{*} \mathrm{u}_{\mathrm{y}}+\mathrm{uu}_{\mathrm{y}}^{*}$

\section{Time Decay of the Local Energy}

Theorem. Assume that the solutions $\mathrm{u}$ and $\mathrm{w}$ are $\mathrm{C}^{3}$ functions such that $|\mathrm{u}|,\left|u_{x}\right|,\left|u_{x x}\right|$ and $|\mathrm{w}|$ all

approach zero as $|\mathrm{x}|$ and $|\mathrm{y}|$ are approaching infinity. Then, given $\mathrm{r}>0, \quad \iint_{M(r)}|u|^{2} d x d y \rightarrow 0$, as $\mathrm{t} \rightarrow \infty$.

where $M(r)=\left\{(x, y) \mid x^{2}+y^{2} \leq r^{2}\right\}$

Proof:

We shall use the method in Lin (1982) to prove this result. Multiplying both sides of (2.1) by a $\mathrm{C}^{3}$ function $\mathrm{A}(\mathrm{x}, \mathrm{y})$ such that $|\mathrm{A}|,\left|A_{x}\right|,\left|A_{y}\right|,\left|A_{x x}\right|$ and $\left|A_{x x x}\right|$ are all bounded, we get

$$
\begin{gathered}
\partial\left(\mathrm{A}|u|^{2}\right) / \partial \mathrm{t}+A_{x}|u|^{2}-A_{x x x}|u|^{2}+3 A_{x}\left|u_{x}\right|^{2}-A_{y} w u^{*}-A_{y} w^{*} \mathrm{u}+A_{x}|w|^{2} \\
=\partial \mathrm{F} / \partial \mathrm{x}+\partial \mathrm{G} / \partial \mathrm{y}
\end{gathered}
$$

where $\mathrm{F}=\mathrm{A}|u|^{2}-\mathrm{A}\left(|u|^{2}\right)_{x}+A_{x}\left(|u|^{2}\right)_{x^{-}} A_{x x}\left(|u|^{2}\right)+3 \mathrm{~A}\left|u_{x}\right|^{2}+A_{x}|\mathrm{w}|^{2}$ and $\mathrm{G}=-\mathrm{Awu}^{*}-\mathrm{Aw}^{*} \mathrm{u}$.

Integrating both sides with respect to the entire $x-y$ space, we get

$$
\partial\left(\iint_{R^{2}} A|u|^{2} d x d y\right) / \partial \mathrm{t}+\iint_{R^{2}}\left[\left(A_{x}-A_{x x x}\right)|u|^{2}+A_{x}|w|^{2}+3 A_{x}\left|u_{x}\right|^{2}-A_{y}\left(w u^{*}+w^{*} u\right)\right] \mathrm{dxdy}=0
$$

Now we integrate both sides with respect to $\mathrm{t}$ from 0 to $\mathrm{T}, \mathrm{T}>0$, to get

$$
\begin{aligned}
& \iint_{0}^{T} \int_{R^{2}}\left[\left(A_{x}-A_{x x x}\right)|u|^{2}+A_{x}|W|^{2}+3 A_{x}\left|u_{x}\right|^{2}-A_{y}\left(w u^{*}+w^{*} u\right)\right] d x d y d t \\
& =\iint_{R^{2}} A|u|^{2}(x, y, 0) d x d y-\iint_{R^{2}} A|u|^{2}(x, y, T) d x d y
\end{aligned}
$$

Using (2.2), we get

$$
\int_{0}^{T} \iint_{R^{2}}\left[\left(A_{x}-A_{x x x}-\left|A_{y}\right|\right)|u|^{2}+\left(A_{x}-\left|A_{y}\right|\right)|w|^{2}+3 A_{x}\left|u_{x}\right|^{2}\right] d x d y d t \leq c_{1}
$$

where $c_{1}$ is a constant depending on the initial data and the bound for A. Note that $c_{1}$ doesn't depend on T.

We now choose $\mathrm{A}(\mathrm{x}, \mathrm{y})=\arctan \left(\frac{x}{3}+\frac{y}{6}\right)$. Then

$$
\left(A_{x}-A_{x x x}-\left|A_{y}\right|\right)>0, A_{x^{-}}\left|A_{y}\right|>0, \text { and } A_{x}>0 .
$$

Let $r>0$. We get

$$
\int_{0}^{\infty} \int_{M(3 r)}\left[|u|^{2}+|w|^{2}+\left|u_{x}\right|^{2}\right] d x d y d t \leq c_{2}
$$


where $c_{2}$ depends on the initial data, $\mathrm{A}$, and $\mathrm{r}$.

Let $\mathrm{B}$ be a $C^{3}$ function which depends only on $\mathrm{x}$ and $\mathrm{y}, \mathrm{B}=0$ if $\mathrm{x}^{2}+\mathrm{y}^{2} \geq 9 r^{2}, \mathrm{~B}=1$ if $\mathrm{x}^{2}+\mathrm{y}^{2} \leq 4 r^{2}$, and $0 \leq \mathrm{B} \leq 1$.

Then

$$
\left|\iint_{M(3 r)} B\left(\partial\left(|u|^{2}\right) / \partial t\right) d x d y\right| \leq c_{3} \iint_{M(3 r)}\left[|u|^{2}+|w|^{2}+\left|u_{x}\right|^{2}\right] d x d y
$$

where $c_{3}$ depends only on the upper bounds for $\left|B_{x}\right|,\left|B_{x x x}\right|$, and $\left|B_{y}\right|$.

Let $0<\mathrm{s}<\mathrm{t}$, then

$$
(t-s) \iint_{M(r)}|u|^{2} d x d y \leq(t-s) \iint_{M(r)} B|u|^{2} d x d y \leq \int_{s}^{t} \iint_{M(3 r)} B|u|^{2} d x d y d z+\int_{s}^{t}(z-s)\left|\iint_{M(3 r)} B \partial\left(|u|^{2}\right) / \partial t d x d y\right| d z
$$

Let $\mathrm{s}=\mathrm{t}-1$, then

$$
\int_{M(r)}|u|^{2} d x d y \leq c_{4} \int_{t-1}^{t} \iint_{M(3 r)}\left[|u|^{2}+|w|^{2}+\left|u_{x}\right|^{2}\right] d x d y d z
$$

for some constant $c_{4}$ which is independent of $\mathrm{t}$.

Thus by (3.1), $\iint_{M(r)}|u|^{2} d x d y$ goes to zero as t goes to infinity. M(r)

\section{Conclusion}

The result of this paper is the first paper in the weakly dispersion in another direction of the system of equations that models the zero-group-dispersion wavelength of a single-mode optical fiber. There remains several problems for the future study of this system of equations such as the well-posedness, special solutions, symmetries, etc. Moreover, we have found two conservation laws. It would be very interesting to find more conservation laws, if any additional conservation law exists.

\section{Acknowledgement}

A part of this work was carried out while the author was visiting Institute of Mathematics, Academia Sinica, Taipei, during May 22 - June 6, 2017. The author wishes to thank Professor Jyh-hao Lee for his generous support and hospitality during the visit. The author also wishes to thank the reviewers of their comments which have greatly improved the content of this article.

\section{References}

Kadomtsev, B. B., \& Petviashvili, V. I. (1970). On the stability of solitary waves in weakly dispersive media. Sov. Phys. Dokl., 15, 539-541

Korteweg, D. J., \& de Vries, G. (1895). On the Change of Form of Long Waves Advancing in a Rectangular Canal, and on a New Type of Long Stationary Waves. Philosophical Magazine, 39(240), 422-443. https://doi.org/10.1080/14786449508620739

Lin, J. (1982). Decay of the local energy of a two-dimensional wave in a nonlinear weakly negative dispersive medium. Physics Letters A, 90 A, 278-279.

Noether, E. (1918). Invariante Variationsprobleme. Nachr. D. König. Gesellsch. D. Wiss. Zu Göttingen, Math-phys. Klasse., 235-257.

Wai, P. K. A., Chen, H. H., \& Lee, Y. C. (1990). Radiations by "Solitons" at the zero group-dispersion wavelength of single-mode optical fibers. Physical Review A, 41, 426 - 439. https://doi.org/10.1103/PhysRevA.41.426

Wai, P. K. A., Menyuk, C. R., Lee, Y. C., \& Chen, H. H. (1986). Nonlinear pulse propagation in the neighborhood of the zero-dispersion wavelength of monomode optical fibers. Optics Letters, 11, 464-466.

https://doi.org/10.1364/OL.11.000464 
Wai, P. K. A., Menyuk, C. R., Chen, H. H., \& Lee, Y. C. (1987). Soliton at the zero-group-dispersion wavelength of a single-model fiber. Optics Letters, 12, 628 - 630. https://doi.org/10.1364/OL.12.000628

\section{Copyrights}

Copyright for this article is retained by the author(s), with first publication rights granted to the journal.

This is an open-access article distributed under the terms and conditions of the Creative Commons Attribution license (http://creativecommons.org/licenses/by/4.0/). 